\title{
A Remark on Diffusion of Directed Polymers in Random Environments
}

\author{
Renming Song ${ }^{1}$ and Xian Yin Zhou ${ }^{2}$
}

Received November 8,1995

\begin{abstract}
We consider a system of random walks or directed polymers interacting with an environment which is random in space and time. Under minimal assumptions on the distribution of the environment, we prove that this system has diffusive behavior with probability one if $d>2$ and $\beta<\beta_{0}$, where $\beta_{0}$ is defined in terms of the probability that the symmetric nearest neighbor random walk on the $d$-dimensional integer lattice ever returns to its starting point. We also obtain a precise estimate for the mean square displacement of this system.
\end{abstract}

KEY WORDS: Random walks; directed polymers; random environment: martingales.

\section{INTRODUCTION AND THE MAIN RESULT}

A directed polymer system is a statistical ensemble of walks or paths in $\mathbb{Z}^{d}$ parametrized by time. The graph of the walk in $\mathbb{Z}^{d+1}$ is the "polymer" which moves at a constant rate in the time direction and so is called "directed." A more formal description of a directed polymer system is as follows. Let $\left\{X_{n}\right\}_{n \geq 0}$ be a simple random walk in $\mathbb{Z}^{d}$ on a probability space $(\Omega, \mathscr{F}, P) .(n, X(n))$ is our model of a directed polymer. Let $P_{x}$ be the probability measure of $\left\{X_{n}\right\}$ starting at $x$, and $E_{x}$ be the corresponding expectation. For convenience, we set $P=P_{0}$ and $E=E_{0}$. The environment is given by $h(n, x), n \in \mathbb{N}, x \in \mathbb{Z}^{d}$, a collection of independent and identically distributed random variables on a probability space $\left(\Omega_{H}, \mathscr{F}_{H}, P_{H}\right)$. We also assume that $h(n, x), n \in \mathbb{N}, x \in \mathbb{Z}^{d}$ are independent of the random walk $X$. We use $E_{H}$ to stand for the expectation with respect to $P_{H}$. The directed

\footnotetext{
' Department of Mathematics, University of Michigan, Ann Arbor, Michigan 48109.

2Institut für Mathematik, Ruhr-Universität Bochum, D-44780 Bochum, Germany, and Department of Mathematics, Beijing Normal University, Beijing 100875, China.
} 
polymer $(n, X(n))$ interacts with the environment $\left\{h(n, x), n \in \mathbb{N}, x \in \mathbb{Z}^{d}\right\}$, producing an interacting density

$$
q(n, x)=E\left(\exp \left[\beta \sum_{j=1}^{n} h(j, X(j))\right] ; X(n)=x\right)
$$

where $\beta>0$ is a constant. This density is unnormalized, and so we normalize it to

$$
q_{N}(n, x)=q(n, x) / Z_{\beta}(n)
$$

where $Z_{\beta}(n)$ is the partition function

$$
Z_{\beta}(n)=\sum_{x} q(n, x)=E\left(\exp \left[\beta \sum_{j=1}^{n} h(j, X(j))\right]\right)
$$

The parameter $\beta$ describes the strength of disorder or the extent to which the directed polymer interacts with the random environment. When $\beta>0$ is small, the interaction is weak, and when $\beta>0$ is large, the interaction is strong. We are interested in studying the asymptotic behavior of the mean square displacement of the interacting system

$$
\begin{aligned}
E_{\beta, n, h}\left(|X(n)|^{2}\right) & =\sum_{x} x^{-2} q_{N}(n, x) \\
& =\frac{E\left(|X(n)|^{2} \exp \left[\beta \sum_{j=1}^{n} h(j, X(j))\right]\right)}{Z_{\beta}(n)}
\end{aligned}
$$

There are many papers dealing in physical terms with the asymptotic behavior of the mean square displacement $E_{\beta, n, n}\left(|X(n)|^{2}\right)$ as $n \rightarrow \infty$. The results in the physics literature are as follows. (i) When $d \leqslant 2$, the directed polymer is superdiffusive in the sense that $E_{\beta, n, h}\left(|X(n)|^{2}\right)$ behaves like $n^{2 \zeta}$ for some $\zeta>1 / 2$. In particular, it is believed that $\zeta=2 / 3$ when $d=1$. (ii) When $d>2$, the directed polymer is diffusive in the sense that $E_{\beta, n, h}\left(|X(n)|^{2}\right)$ behaves like $n$ as $n \rightarrow \infty$ if $\beta>0$ is sufficiently small, and it is superdiffusive if $\beta>0$ is sufficiently large. The arguments in the physics literature are either heuristic or numerical; they are not mathematically rigorous.

The first rigorous result concerning the asymptotic behavior of $E_{\beta, n, h}\left(|X(n)|^{2}\right)$ is due to Imbrie and Spencer. They proved in ref. 6 that when $d>2$, if $\beta>0$ is small enough, and if for any $n \in \mathbb{N}$ and $x \in \mathbb{Z}^{d}$, $h(n, x)= \pm 1$ with probability $1 / 2$ each, then for typical realizations of the 
environment, the directed polymer system is diffusive, in other words the following holds with probability one:

$$
\lim _{n \rightarrow \infty} \frac{E_{\beta, n, n}\left(|X(n)|^{2}\right)}{n}=1
$$

They actually obtained in ref. 6 a more precise estimate than (1). Later, Bolthausen ${ }^{(2)}$ used a martingale approach to prove a central limit theorem for the system given in ref. 6 . By means of the martingale approach, Albeverio and Zhou ${ }^{(1)}$ recently obtained a Wiener process behavior and a precise mean square displacement for the system given in ref. 6. However, the coupling constants $\beta>0$ given in refs. 1,2 , and 6 are assumed to be sufficiently small. There is no rigorous result concerning the asymptotic behavior of $E_{\beta, \ldots .1}\left(|X(n)|^{2}\right)$ when $d \leqslant 2$.

It seems that the assumption that $h(n, x)= \pm 1$ with probability $1 / 2$ each is probably not the assumption physicists had in mind. This is because Olsen and Song ${ }^{(9)}$ proved that when $d \geqslant 4$, if $h(n, x)= \pm 1$ with probability $1 / 2$ each, then ( 1 ) is always true with probability 1 for all $\beta>0$, contradicting the results in the physics literature. The assumption physicists had in mind about the distribution of the environment is probably that $h$ follows a continuous distribution, like the normal distribution or a uniform distribution.

In this paper we study the asymptotic behavior of random directed polymers under the following more general assumption on the environment variables $h(n, x)$ :

$$
E_{H} \exp [\gamma h(n, x)]<\infty, \quad \forall \gamma>0
$$

Normal distributions and uniform distributions certainly satisfy the above assumption.

Our way of approaching the problem above is that we first ignore the coupling constant $\beta$ and assume that $\beta=1$. For convenience, put $Z(n)=Z_{1}(n)$ and $E_{n, h}=E_{1, n, h}$. Let

$$
H(n, x)=\frac{\exp [h(n, x)]-E_{H} \exp [h(n, x)]}{E_{H} \exp [h(n, x)]}
$$

and put $\lambda=E_{H}\left(H(n, x)^{2}\right)$. In this paper we prove the following result:

Theorem 1. Suppose that $d>2$. If

$$
\lambda<\frac{1-p_{d}}{p_{d}}
$$


then with probability 1 ,

$$
\lim _{n \rightarrow \infty} \frac{E_{n, h}\left(|X(n)|^{2}\right)}{n}=1
$$

where $p_{c}$ is the probability that the random walk $X(n)$ ever returns to its starting point,

$$
p_{d}=P(X(i)=0 \text { for some } i>0)
$$

Sinai "11) also studied the diffusive behavior of the above system. Under the same assumption as in Theorem 1, Sinai ${ }^{(1)}$ obtained a diffusive behavior (in the weak sense) for this system. Here we prove that this system has diffusive behavior with probability one. A more precise form of Theorem 1 will be given in the next section (see Theorem 2 below). Moreover, we will still use the martingale approach to prove our main result.

It would be very interesting if one could find the "critical" value $\lambda_{c}$. such that when $\lambda<\lambda_{\text {c }}$ the conclusion of Theorem 1 is true, while when $\lambda>\lambda_{\text {c }}$ the conclusion of Theorem 1 is not. We have not been able to do that yet. However, Theorem 1 tells us that $\lambda_{0}=\left(1-p_{d}\right) / p_{d}$ is a lower bound for $\lambda_{c}$. It is known that $p_{d} \rightarrow 0$ as $d \rightarrow \infty$, thus $\lambda_{0} \rightarrow \infty$ as $d \rightarrow \infty$. We also know that $p_{3}=0.340537 \cdots$ (see, for instance, ref. 12). For numerical values of $p_{d}$ for $4 \leqslant d \leqslant 20$ and some other values of $d$, see ref. 3 .

Theorem 1 can be easily translated into the language of random directed polymers. For instance, the following result is a direct consequence of Theorem 1.

Corollary 1. Suppose $d>2$ and that for any $n \in \mathbb{N}$ and $x \in \mathbb{Z}^{d}$, $h(n, x)$ follows the standard normal distribution. If $\beta<\left(-\ln p_{d}\right)^{1 / 2}$, then for typical realizations of the environment $h$, our directed polymer system is diffusive, or more precisely

$$
\lim _{n \rightarrow \infty} \frac{E_{\beta, n, h}\left(|X(n)|^{2}\right)}{n}=1
$$

with probability 1 .

Proof. If we replace $h(n, x)$ by $\beta h(n, x)$ in Theorem 1 , then under our assumption that $h(n, x)$ is a standard normal random variable, we have

$$
\lambda=e^{\beta^{2}}-1
$$

The inequality $\lambda<\left(1-p_{d}\right) / p_{d}$ is equivalent to $\beta<\left(-\ln p_{d}\right)^{1 / 2}$. QED 
As a consequence of Theorem 1 , we can easily get the following result, which is a generalization of the main result of ref. 9 .

Corollary 2. Suppose $d \geqslant 3$ and that for any $n \in \mathbb{N}$ and $x \in \mathbb{Z}^{d}$, $h(n, x)= \pm 1$ with probability $1 / 2$ each. Then for any $\beta>0$, we have

$$
\lim _{n \rightarrow \infty} \frac{E_{\beta, n, h}\left(|X(n)|^{2}\right)}{n}=1
$$

with probability 1 , i.e., for typical realizations of the environment $h$, our directed polymer system is diffusive.

Proof. If we replace $h(n, x)$ by $\beta h(n, x)$ in Theorem 1 , then under our assumption about the environment,

$$
\lambda=\frac{\cosh (2 \beta)-\cosh ^{2} \beta}{\cosh ^{2} \beta}<1
$$

while we know that $\left(1-p_{d}\right) / p_{d}$ is always larger than 1 when $d \geqslant 3$. QED

\section{PROOF OF THE MAIN RESULT}

In this section we prove our main result. First we need to introduce some notations and establish some preliminary results.

Let $r_{i}=P(X(2 i)=0)$ be the probability that the random walk returns to its starting point at the $2 i$ th step. Then it is known that

$$
\sum_{i=1}^{\infty} r_{i}=\frac{p_{d}}{1-p_{d}}
$$

(see, for instance, ref. 10). We use this identity several times in the argument below.

It is clear that

$$
\exp \left[\sum_{j=1}^{n} h(j, X(j))\right]=\left\{E_{H} \exp [h(1,0)]\right\}^{n} \prod_{j=1}^{n}[1+H(j, X(j))]
$$

so if we put

then we have

$$
\kappa(n)=E \prod_{j=1}^{n}[1+H(j, X(j))]
$$

$$
Z(n)=\left\{E_{H} \exp [h(1,0)]\right\}^{n} \kappa(n)
$$


and

$$
E_{n, h}\left(|X(n)|^{2}\right)=\frac{E\left(|X(n)|^{2} \prod_{j=1}^{n}[1+H(j, X(j))]\right)}{\kappa(n)}
$$

Let us first prove two lemmas.

Lemma 1. For $d>2$, if

$$
\lambda<\frac{1-p_{d}}{p_{d}}
$$

then $\kappa(n)$ converges almost surely to a random variable $\zeta$ satisfying

$$
E_{H} \zeta=1 \quad \text { and } \quad P_{H}(\zeta=0)=0
$$

Proof. It is easy to see that $\{\kappa(n)\}_{n \geqslant 1}$ is a $P_{H}$-martingale with respect to the $\sigma$-fields $\mathscr{F}_{n}$ generated by the variables $h(m, x), m \leqslant n, x \in \mathbb{Z}^{d}$. Therefore $\kappa(n)$ converges almost surely by the martingale limit theorem, say, to $\zeta$.

Let $\{Y(n)\}_{n \geqslant 0}$ be an independent copy of $\{X(n)\}_{n \geqslant 0}$. We assume that $\{Y(n)\}_{n \geq 0}$ is also independent of the environment. Then

$$
\begin{aligned}
E_{H}\left(\kappa(n)^{2}\right) & =E_{H}\left(E \prod_{j=1}^{n}[1+H(j, X(j))][1+H(j, Y(j))]\right) \\
& =E\left(E_{H} \prod_{j=1}^{n}[1+H(j, X(j))][1+H(j, Y(j))]\right) \\
& =E \prod_{j=1}^{n}\left(1+\lambda 1_{\{X(j)=Y(j)\}}\right) \\
& =1+\sum_{k=1}^{n} \lambda^{k} \sum_{k \leqslant i_{1}<\cdots<i_{k} \leqslant n} P\left(X\left(i_{1}\right)=Y\left(i_{1}\right), \ldots, X\left(i_{k}\right)=Y\left(i_{k}\right)\right) \\
& =1+\sum_{k=1}^{n} \lambda^{k} \quad \sum_{1 \leqslant i_{1}<\cdots<i_{k} \leqslant n} r_{i_{1}} r_{i_{2}-i_{1}} \cdots r_{i_{k}-i_{k-1}} \\
& \leqslant 1+\sum_{k=1}^{n} \lambda^{k}\left(\sum_{j=1}^{\infty} r_{i}\right)^{k} \\
& =1+\sum_{k=1}^{n}\left(\lambda \frac{p_{d}}{1-p_{d}}\right)^{k}
\end{aligned}
$$


where we used (2). Therefore under the assumption $\lambda<\left(1-p_{d}\right) / p_{d}$, we know that

$$
\sup E_{H}\left(\kappa(n)^{2}\right)<\infty
$$

hence we can conclude that $\kappa(n)$ converges to $\zeta$ in $L^{1}$ and $L^{2}$. Therefore $E_{H}(\zeta)=1$ and $P_{H}(\zeta=0)<1$. It is easy to see that the event $\{\zeta=0\}$ belongs to the tail field

$$
\bigcap_{n} \sigma\left(h(m, x): m \geqslant n, x \in \mathbb{Z}^{d}\right)
$$

and thus by Kolmogorov's zero-one law we know that $P_{H}(\zeta=0)=0$. QED Let

$$
f_{n}=E \prod_{i=1}^{n}[1+H(i, X(i))]\left(\left|X_{n}\right|^{2}-n\right), \quad \forall n \geqslant 1
$$

Then it is easy to check that $\left\{f_{n}\right\}_{n \geqslant 1}$ is a $P_{H}$ martingale with respect to the filtrations $\mathscr{F}_{n}$.

Lemma 2. For $d>2$, if $\lambda<\left(1-p_{d}\right) / p_{d}$, then there is a constant $C(d) \in(0, \infty)$ such that

$$
E_{H} f_{n}^{2} \leqslant \begin{cases}C(d), & d>6 \\ C(d) \log n, & d=6 \\ C(d) n^{3-d / 2}, & 2<d<6\end{cases}
$$

Proof. Again let $\{Y(n)\}_{n \geq 0}$ be an independent copy of $\{X(n)\}_{n \geqslant 0}$, and assume that $\{Y(n)\}_{n \geqslant 0}$ is also independent of the environment. Then

$$
\begin{aligned}
E_{H}\left(f_{n}^{2}\right) & =E_{H} E\left(\prod_{i=1}^{n}\left[1+H\left(i, X_{i}\right)\right]\left[1+H\left(i, Y_{i}\right)\right]\left(\left|X_{n}\right|^{2}-n\right)\left(\left|Y_{n}\right|^{2}-n\right)\right) \\
& =E\left(\prod_{i=1}^{n}\left(1+\lambda \sigma 1_{\left\{x_{i}=Y_{i}\right\}}\right)\left(\left|X_{n}\right|^{2}-n\right)\left(\left|Y_{n}\right|^{2}-n\right)\right) \\
& =\sum_{k=1}^{n} \lambda^{k} \sum_{1 \leqslant i_{1}<\cdots<i_{k} \leqslant n} E\left(\left|X_{n}\right|^{2}-n\right)\left(\left|Y_{n}\right|^{2}-n\right) 1_{\left\{X_{i_{1}}=Y_{i_{1}} \ldots, x_{i_{k}}=Y_{\left.i_{k}\right\}}\right.} \\
& =\sum_{k=1}^{n} \lambda^{k} \sum_{1 \leqslant i_{1}<\cdots<i_{k} \leqslant n} E\left(\left|X_{i_{k}}\right|^{2}-i_{k}\right)\left(\left|Y_{i_{k}}\right|^{2}-i_{k}\right) 1_{\left\{x_{i_{1}}=Y_{i_{1}} \ldots, x_{i_{k}}=Y_{\left.i_{k}\right\}}\right\}} \\
& =\sum_{k=1}^{n} \lambda^{k} \sum_{1 \leqslant i_{1}<\cdots<i_{k} \leqslant n} E\left(\left|X_{i_{k}}\right|^{2}-i_{k}\right)^{2} 1_{\left\{x_{i_{1}}=Y_{i_{1}}, \ldots . x_{i_{k}}=Y_{\left.i_{k}\right\}}\right\}}
\end{aligned}
$$


Noting that

$$
\begin{aligned}
\left|X_{i_{k}}\right|^{2}-i_{k}= & \sum_{j=1}^{k}\left(\left|X_{i_{j}}-X_{i_{j}-1}\right|^{2}-\left(i_{j}-i_{j-1}\right)\right) \\
& +2 \sum_{1 \leqslant j_{1}<j_{2} \leqslant k}\left[X\left(i_{j_{2}}\right)-X\left(i_{j_{2}-1}\right)\right]\left[X\left(i_{j_{1}}\right)-X\left(i_{j_{1}-1}\right)\right]
\end{aligned}
$$

we get that

$$
\begin{aligned}
\left(\left|X_{i_{k}}\right|^{2}-\right. & \left.i_{k}\right\}^{2} \\
\leqslant & 2\left\{\sum_{j=1}^{k}\left[\left|X_{i_{j}}-X_{i_{j-1}}\right|^{2}-\left(i_{j}-i_{j-1}\right)\right]\right\}^{2} \\
& +2\left\{2 \sum_{1 \leqslant j_{1}<j_{2} \leqslant k}\left[X\left(i_{j_{2}}\right)-X\left(i_{j_{2}-1}\right)\right]\left[X\left(i_{j_{j}}\right)-X\left(i_{j_{1}-1}\right)\right]\right\}^{2} \\
\leqslant & 2\left\{\sum_{j=1}^{k}\left[\left|X_{i_{j}}-X_{i_{j-1}}\right|^{2}-\left(i_{j}-i_{j-1}\right)\right]\right\}^{2} \\
& +2(k-1)^{2}\left(\sum_{j=1}^{k}\left|X_{i_{j}}-X_{i_{j-1}}\right|^{2}\right)^{2} \\
\leqslant & 2 k^{2} \sum_{j=1}^{k}\left[\left|X_{i_{j}}-X_{i_{j-1}}\right|^{2}-\left(i_{j}-i_{j-1}\right)\right]^{2} \\
& +2(k-1)^{2} k^{2} \sum_{j=1}^{k}\left|X_{i_{j}}-X_{i_{j-1}}\right|^{4} \\
\leqslant & 2 k^{2}\left[1+2(k-1)^{2}\right] \sum_{j=1}^{k}\left[\left|X_{i_{j}}-X_{i_{j-1}}\right|^{2}-\left(i_{j}-i_{j-1}\right)\right]^{2} \\
& +4(k-1)^{2} k^{2} \sum_{j=1}^{k}\left(i_{j}-i_{j-1}\right)^{2}
\end{aligned}
$$

Therefore $E_{H}\left(f_{n}^{2}\right)$ is less than or equal to

$$
\begin{aligned}
& \sum_{k=1}^{n} 2 k^{2}\left[1+2(k-1)^{2}\right] \lambda^{k} \\
& \quad \times \sum_{1 \leqslant i_{1}<\cdots<i_{k} \leqslant n} \sum_{j=1}^{k} E\left(\left[\left|X_{i_{j}}-X_{i_{j-1}}\right|^{2}-\left(i_{j}-i_{j-1}\right)\right]^{2}\right. \\
& \quad \times 1_{\left\{x_{i_{1}}=Y_{i_{1}} \ldots . . x_{i_{k}}=Y_{\left.i_{k}\right\}}\right\}}
\end{aligned}
$$




$$
\begin{aligned}
& +\sum_{k=1}^{n} 4(k-1) 2 k^{2} \lambda^{k} \sum_{1 \leqslant i_{1}<\cdots<i_{k} \leqslant n} \sum_{j=1}^{k}\left(i_{j}-i_{j-1}\right)^{2} \\
& \times P\left(X\left(i_{1}\right)=Y\left(i_{1}\right), \ldots, X\left(i_{k}\right)=Y\left(i_{k}\right)\right) \\
= & I_{1}+I_{2}
\end{aligned}
$$

It is easy to see that

$$
\begin{aligned}
I_{2} & =\sum_{k=1}^{n} 4(k-1) 2 k^{2} \lambda^{k} \sum_{1 \leqslant i_{1}<\cdots<i_{k} \leqslant n} \sum_{j=1}^{k} r_{i_{1}} r_{i_{2}-i_{1}} \cdots r_{i_{k}-i_{k-1}}\left(i_{j}-i_{j-1}\right)^{2} \\
& \leqslant \sum_{k=1}^{n} 4(k-1) 2 k^{3} \lambda^{k}\left(\sum_{i=1}^{\infty} r_{i}^{k-1}\right)\left(\sum_{i=1}^{n} i^{2} r_{i}\right) \\
& \leqslant \begin{cases}O(1), & d>6 \\
O(1) \log n, & d=6 \\
O(1) n^{3-d / 2}, & 2<d<6\end{cases}
\end{aligned}
$$

where we used the fact that when $i$ is large

$$
r_{i} \sim 2\left(\frac{d}{4 i \pi}\right)^{d / 2}
$$

Now we estimate $I_{1}$. Note that for $1 \leqslant j \leqslant k$,

$$
\begin{aligned}
& E\left(\left[\left|X_{i_{j}}-X_{i_{j-1}}\right|^{2}-\left(i_{j}-i_{j-1}\right)\right]^{2} 1_{\left\{X_{i_{1}}=Y_{i_{1}} \ldots, X_{i_{k}}=Y_{i_{k}}\right\}}\right) \\
& =r_{i_{j+1}-i_{j}} \cdots r_{i_{k}-i_{k-1}} E\left\{1_{\left\{X\left(i_{1}\right)=r\left(i_{1}\right) \ldots . . X\left(i_{j-1}\right)=r\left(i_{j-1}\right)\right\}}\right. \\
& \left.\times E\left(\left[\left|X\left(i_{j}-i_{j-1}\right)\right|^{2}-\left(i_{j}-i_{j-1}\right)\right]^{2} 1_{\left\{X\left(i_{j}-i_{j-1}\right)=Y\left(i_{j}-i_{j-1}\right)\right\}}\right)\right\} \\
& \leqslant r_{i_{j+1}-i_{j}} \cdots r_{i_{k}-i_{k-1}} E\left\{1_{\left\{X\left(i_{1}\right)=Y\left(i_{1}\right) \ldots \ldots . X\left(i_{j-1}\right)=y\left(i_{j-1}\right)\right\}}\right. \\
& \times \sum_{x} E\left(\left[\left|X\left(i_{j}-i_{j-1}\right)\right|^{2}-\left(i_{j}-i_{j-1}\right)\right]^{2}\right. \\
& \left.\left.\times 1_{\left\{X\left(i_{j}-i_{j-1}\right)=x\right\}} P\left(Y\left(i_{j}-i_{j-1}\right)=x\right)\right)\right\} \\
& \leqslant O(1) r_{i_{j+1}-i_{j}} \cdots r_{i_{k}-i_{k-1}}\left(i_{j}-i_{j-1}\right)^{-d / 2} \\
& \times E\left\{1_{\left\{X\left(i_{1}\right)=Y\left(i_{1}\right) \ldots . . . X\left(i_{j-1}\right)=Y\left(i_{j-1}\right)\right\}} E\left(\left[\left|X\left(i_{j}-i_{j-1}\right)\right|^{2}-\left(i_{j}-i_{j-1}\right)\right]^{2}\right)\right\} \\
& \leqslant O(1) r_{i_{1}} \cdots r_{i_{j-1}-i_{j-2}} r_{i_{j+1}-i_{j}} \cdots r_{i_{k}-i_{k-1}}\left(i_{j}-i_{j-1}\right)^{2-d / 2}
\end{aligned}
$$


where we have used that facts that

$$
E\left(\left[|X(i)|^{2}-i\right]^{2}\right) \leqslant O(1) i^{2}
$$

and

$$
P\left(X_{i}=x\right) \leqslant O(1) i^{-d / 2}
$$

Therefore we have

$$
\begin{aligned}
I_{1} \leqslant & O(1) \sum_{k=1}^{n} 2 k^{3}\left[1+2(k-1)^{2}\right] \lambda^{k}\left(\sum_{i=1}^{\infty} r_{i}\right)^{k-1} \sum_{i=1}^{n} i^{2-d / 2} \\
& \leqslant \begin{cases}O(1), & d>6 \\
O(1) \log n, & d=6 \\
O(1) n^{3-d / 2}, & 2<d<6\end{cases}
\end{aligned}
$$

The proof is now complete. QED

Remark 1. By modifying the above argument, we can actually prove that if $0<\lambda<\left(1-p_{d}\right) / p_{d}$, then there is a constant $c_{d} \in(0, \infty)$ such that:

(i) $\lim _{n \rightarrow \infty} E_{H} f_{n}^{2}=c_{d}$ for $d>6$.

(ii) $\lim _{n \rightarrow \infty} E_{H} f_{n}^{2} / \log n=c_{d}$ for $d=6$.

(iii) $\lim _{n \rightarrow \infty} n^{-3+d / 2} E_{H} f_{n}^{2}=c_{d}$ for $2<d<6$.

The following theorem is an improvement of Theorem 1.

Theorem 2. Assume $\lambda<\left(1-p_{d}\right) / p_{d}$.

(i) If $d>6$, then there is a finite random variable $\xi$ such that the following limit holds with probability 1 :

$$
\lim _{n \rightarrow \infty}\left(E_{n, h}\left|X_{n}\right|^{2}-n\right)=\xi
$$

(ii) For $d=6$, for any given $\delta \in(0,1)$ there is a finite random variable $\eta$ such that the following holds with probability 1 :

$$
\left.\left|E_{n, h}\right| X_{n}\right|^{2}-n \mid \leqslant \eta(\log n)^{1 / 2+\delta}, \quad \forall n \geqslant 2
$$

(iii) For $2<d<6$, for any given $\delta \in(0,1)$ there is also a finite random variable $\eta$ such that the following holds with probability 1 :

$$
\left.\left|E_{n, h}\right| X_{n}\right|^{2}-n \mid \leqslant \eta n^{3 / 2-d / 4+\delta}, \quad \forall n \geqslant 1
$$


Proof. The proof of this theorem is similar to that of Theorem 1.1 of ref. 1. We provide the proof of (iii) here for the reader's convenience.

Set $a_{n}=n^{1 / \delta}, \forall n \geqslant 1$. By Lemma 2 we can show that

$$
\begin{aligned}
E_{H}\left(\left|f_{a_{n}}\right| 1_{\left\{\left|f_{u_{n}}\right|>a_{n}^{3 / 2-d / 4+s}\right\}}\right) & \leqslant a_{n}^{-3 / 2+d / 4-\delta} E_{H}\left(\left|f_{a_{n}}\right|^{2}\right) \\
& \leqslant O(1) a_{n}^{-3 / 2+d / 4-\delta} a_{n}^{3-d / 2} \\
& =O(1) a_{n}^{3 / 2-d / 4-\delta}
\end{aligned}
$$

Thus by Theorem 2.4 on p. 16 of ref. 4 we have that

$$
\begin{aligned}
P_{H}\left(\max _{1 \leqslant i \leqslant a_{n}}\left|f_{i}\right|>2 a_{n}^{3 / 2-d / 4+\delta}\right) & \leqslant a_{n}^{-3 / 2+d / 4-\delta} E_{H}\left(\left|f_{a_{n}}\right| 1_{\left\{\left|f_{a_{n}}\right|>a_{n}^{3 / 2-d / 4+\delta}\right\}}\right) \\
& \leqslant O(1) a_{n}^{-3 / 2+d / 4-\delta} a_{n}^{3 / 2-d / 4-\delta} \\
& =O(1) a_{n}^{-2 \delta}=O(1) n^{-2}
\end{aligned}
$$

Let

$$
\Omega_{0}=\left\{\bigcup_{m=1}^{\infty} \bigcap_{n=m}^{\infty}\left\{\max _{1 \leqslant i \leqslant a_{n}}\left|f_{i}\right| \leqslant 2 a_{n}^{3 / 2-d / 4+\delta}\right\}\right\}
$$

Then using the Borel-Cantelli lemma, we can get that $P_{H}\left(\Omega_{0}\right)=1$. It is easy to see that when $n$ is large,

$$
a_{n-1}^{3 / 2-d / 4+\delta} \geqslant \frac{1}{2} a_{n}^{3 / 2-d / 4+\delta}
$$

For each $\omega \in \Omega_{0}$, there is $N(\omega)>1$ such that

$$
\max _{1 \leqslant i \leqslant a_{n}}\left|f_{i}\right| \leqslant 2 a_{n}^{3 / 2-d / 4+\delta}, \quad \forall n \geqslant N(\omega)
$$

If $i \geqslant a_{N(\omega)}$, there is $N_{1}(\omega) \geqslant N(\omega)$ such that

$$
a_{N_{1}(\omega)} \leqslant i<a_{N_{1}(\omega)+1}
$$

and so

$$
\begin{aligned}
\left|f_{i}\right| & \leqslant \max _{1 \leqslant j \leqslant a_{N_{1}(\omega)+1}}\left|f_{j}\right| \\
& \leqslant 2 a_{N_{1}(\omega)+1}^{3 / 2-d / 4+\delta} \\
& \leqslant 4 a_{N_{1}(\omega)}^{3 / 2-d / 4+\delta} \\
& \leqslant 4 i^{3 / 2-d / 4+\delta}
\end{aligned}
$$


In other words, for each $\omega \in \Omega_{0}$, there is $N(\omega)>1$ such that

$$
\left|f_{i}\right| \leqslant 4 i^{3 / 2-a / 4+\delta}, \quad \forall i \geqslant a_{N(\omega)}
$$

Let $\widetilde{\Omega}_{0} \subset \Omega_{H}$ be the collection of $\omega$ such that for each $\omega \in \Omega_{0}$,

$$
\lim _{n \rightarrow \infty} \kappa(n)=\zeta>0
$$

Then we know from Lemma 1 that $P_{H}\left(\tilde{\Omega}_{0}\right)=1$. From Lemma 1 we also know that for each $\omega \in \widetilde{\Omega}_{0}$, there is $M(\omega) \geqslant 1$ such that

$$
\zeta(i) \geqslant \frac{1}{2} \zeta, \quad \forall i \geqslant M(\omega)
$$

Thus for each $\omega \in \widetilde{\Omega}_{0} \cap \Omega_{0}$,

$$
\left.\left|E_{i, h}\right| X_{i}\right|^{2}-i \mid=\frac{\left|f_{i}\right|}{\kappa(i)} \leqslant \frac{8}{\zeta} i^{3 / 2-d / 4+\delta}, \quad \forall i \geqslant a_{N(\omega)} \vee M(\omega)
$$

which proves (iii).

Remark 2. As in ref. 1, we can also get a Wiener process behavior with probability one for our system with $\lambda<\left(1-p_{d}\right) / p_{d}$.

\section{ACKNOWLEDGMENT}

One of us (X.Y.Z.) gratefully acknowledges the financial support of the DFG research project.

\section{REFERENCES}

1. S. Albeverio and X. Y. Zhou, A martingale approach to directed polymers in a random environment, J. Theor. Prob. (1996), to appear.

2. E. Bolthausen, A note on the diffusion of directed polymers in a random environment, Commun. Math. Phys. 123:529-534 (1989).

3. P. Griflin, Accelerating beyond the third dimension: Returning to the origin in simple random walk, Math. Sci. 15:24-35 (1990).

4. P. Hall and C. C. Heyde, Martingale Limit Theory and Its Application (Academic Press, New York, 1980).

5. D. A. Huse and C. L. Henly, Pinning and roughening of domain walls in Ising systems due to random impurities, Phys. Rev. Lett. 54:2708-2711 (1985).

6. J. Z. Imbrie and T. Spencer, Diffusion of directed polymers in a randon environment, J. Stat. Phys. 52:609-626 (1988).

7. M. Kardar, Roughening by impurities at finite temperatures, Phys. Rev. Lett. 55:2923 (1985).

8. M. Kardar and Y. C. Zhang, Scaling of directed polymers in random media, Phys. Rev. Lett. 58:2087-2090 (1987). 
9. P. Olsen and R. Song, Diffusion of directed polymers in a strong random environment, J. Stat. Pltys. 83:727-738 (1996).

10. P. Révész, Random Walk in Random and Non-random Environments (World Scientific, Singapore, 1990).

11. Y. Sinai, A note concerning random walks with random potentials, Fund. Math. 147:173-180 (1995).

12. F. Spitzer, Principle of Random Walk, 2nd ed. (Springer-Verlag, Berlin, 1976).

Commanicated by J. L. Lebowit: 